\title{
Cell and Molecular Biology Underpinning the Effects of PEDF on Cancers in General and Osteosarcoma in Particular
}

\author{
Vijay Chandolu and Crispin R. Dass \\ School of Biomedical and Health Sciences, Victoria University, Building 6, St Albans, VIC 3021, Australia \\ Correspondence should be addressed to Crispin R. Dass, crispin.dass@vu.edu.au \\ Received 24 October 2011; Revised 9 March 2012; Accepted 23 March 2012 \\ Academic Editor: Susan E. Crawford
}

Copyright ( $) 2012$ V. Chandolu and C. R. Dass. This is an open access article distributed under the Creative Commons Attribution License, which permits unrestricted use, distribution, and reproduction in any medium, provided the original work is properly cited.

Cancer is becoming an increasingly common disease in which abnormal cells aggressively grow, invade, and metastasize. In this paper, we review the biological functions of PEDF (pigmented epithelium-derived factor) against cancer, with a focus on a particular type of bone cancer called osteosarcoma. PEDF is a $50 \mathrm{kDa}$ glycoprotein and is a potent inhibitor of angiogenesis, via its ability to decrease proliferation and migration of endothelial cells. This paper critically examines the anticancer activities of PEDF via its role in antiangiogenesis, apoptosis-mediated tumor suppression, and increased tumor cell differentiation. Recently, an orthotopic model of osteosarcoma was used to show that treatment with PEDF had the greatest impact on metastases, warranting an evaluation of PEDF efficacy in other types of cancers.

\section{Introduction}

Cancer is characterized by uncontrolled growth and spread of cells harboring some form of genetic aberration. Formation of tumours requires sequential acquisition of defects that endow tumour cells with the ability to grow, invade, and eventually metastasize. Even after significant improvements in surgery, chemotherapy, and radiotherapy, there are still numerous tumours that are unaffected by therapy. By the time a tumour is diagnosed, it has almost certainly begun metastasizing. In such cases radiation therapy and chemotherapy are often used in combination with surgery in the hope that the tumour and its metastases are effectively controlled [1]. Targeted biological therapies, which are safer, are being studied and clinical trials are conducted to gauge efficacy. The main objective of targeted therapy is to attack specific pathways and tumour growth mechanisms without the side effects attached to small molecule drugs and radiotherapy $[2,3]$.

PEDF is a $50-\mathrm{kDa}$ glycoprotein, which is a member of the serine protease inhibitor (serpin) family, and it has multifunctional properties [4]. It is found to be a potent inhibitor of angiogenesis, proliferation and migration of endothelial cells, retinal vascular permeability, and tumour activity [5].
PEDF is responsible for the antiangiogenesis in various ocular compartments [6]. These significant antiangiogenic properties led the scientists to shift focus on to studies examining the potential antitumour activities of PEDF. PEDF expression changes in the course of progression of different tumour types [5]. Researchers did a number of studies and showed that there is opposite relation between PEDF levels, grade and metastatic potential of prostate tumours [7], pancreatic adenocarcinoma [8], prostate, melanoma, ovarian, osteosarcoma, glioma [4], hepatocellular carcinoma [9], and Wilm's tumours [10].

The human PEDF gene has been mapped, and it encodes a 418 amino acid protein [11]. It has an asymmetrical charge distribution, with a high density of basic residues concentrated on one side and of acidic residues on the opposite side. Negatively charged acidic PEDF binds to collagen, lacks neurotrophic activity, and may confer antiangiogenic properties (Figure 1). Mutational studies have shown that positively charged amino acids and negatively-charged amino acids are responsible for heparin and collagen binding, respectively $[12,13]$. There are three phosphorylation sites identified which collectively induce antiangiogenic and neurotrophic activities. A 34-mer peptide and 44-mer peptide are the two major functional epitopes identified (as shown in Figure 2) 


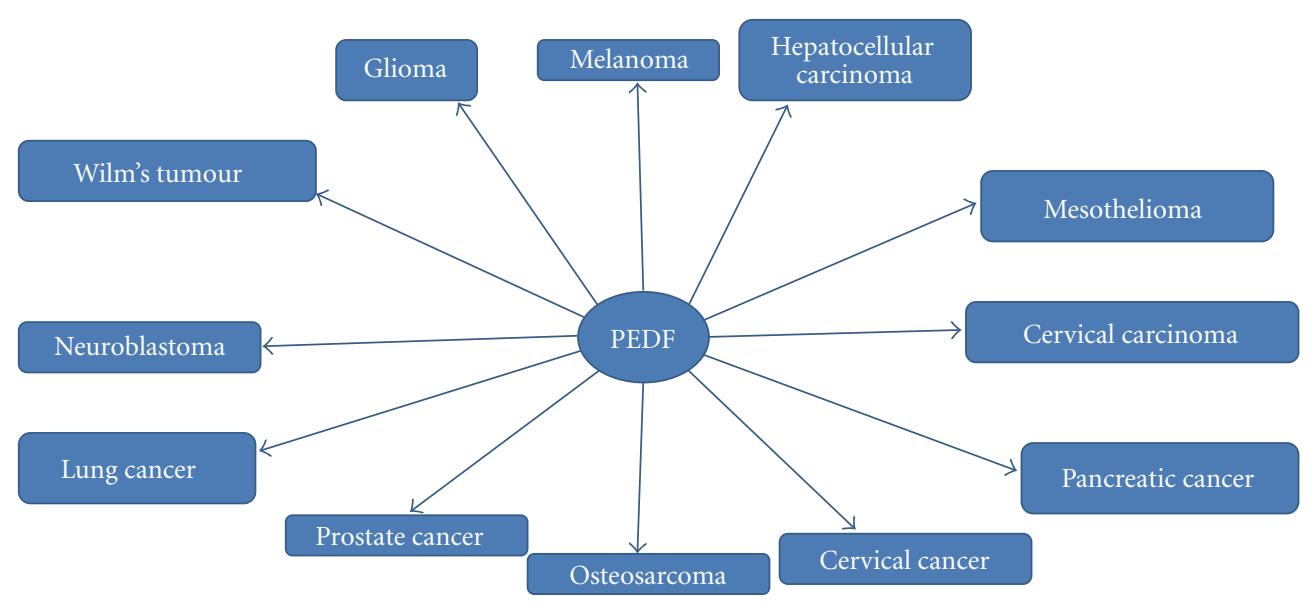

Figure 1: Schematic diagram displaying the functional domains of PEDF. (1) Phosphorylation zones-induce different degrees of antiangiogenic and neurotrophic activity, (2) 34-mer peptide region which has antiangiogenic, blocks cell migration, and induces tumour cell apoptotic activity, (3) 44-mer peptide region which has neurotrophic and cell differentiation activity, (4) basic region (positive) which is necessary for heparin binding, and (5) acidic region (negative) which is responsible for collagen binding and lacks neurotrophic activity.

to date. The 34-mer peptide induces apoptosis, blocks endothelial cell migration and corneal angiogenesis, whereas the 44-mer peptide displays neurotrophic function and the ability to block vascular leakage.

\section{Why Use PEDF for Cancer?}

PEDF has multiple effects against various types of tumour cells as will be outlined below. Furthermore, PEDF is known to inhibit the proliferation and migration of endothelial cells (ECs) and can also promote apoptosis in ECs [5, 18]. Angiogenesis plays a significant role in cancer progression, promoting growth and metastasis of tumours. Inhibition of the signals that assist new blood vessel growth in tumour(s) has become an important target in cancer therapy $[2,3]$. The regulation of angiogenesis involves an inverse relationship between proangiogenic factors and antiangiogenic factors [19]. PEDF not only reduces angiogenesis, but also can increase tumour cell apoptosis and differentiation $[5,18,20]$. These separate functions will be examined in more detail in turn following the next few introductory sections.

\section{Cancer Biology - Initiation, Maintenance, and Progression}

Cancer is the second biggest cause of death in the USA, next to heart disease [1]. In individuals aged 55 years or above, $78 \%$ of all the cancers are being diagnosed. These cancers involve malfunction of genes which control cell growth and division, and only $5 \%$ of all the cancers are hereditary. Approximately 11.4 million Americans with a history of cancer were alive in January 2006, according to The National Cancer Institute. In 2010, about 1,529,560 new cancer cases were predicted to be diagnosed, out of which 789,620 are males and 739,940 are females. More than 1500 people per day were expected to die in 2010.
Cancer is a clonal disease which can initiate from a single cell harboring several genetic mutations. Until recently, very little importance was given to the nature of the cell in which the initiating mutation occurred in human cancer [21]. In recent years, it has emerged that perhaps stem cells are the cells of origin for several types of cancer and studies suggest that a stem cell constitutes the target cell in an increasing number of human solid tumours. In favour of the above notion is the finding that not only some leukaemias but also epithelial tumours can originate in normal stem cells (NSCs), and that carcinomas arise after the accumulation of multiple oncogenic events acquired after long periods of time [21].

The majority of cancers occur as a result of alterations in oncogenes. Among these, gene mutation, amplification, or overexpression of HER2/Neu (ErbB2) and epidermal growth factor receptor (EGFR, ErbB1) have been found in various human cancers [22]. Oncogenes encode proteins that control cell proliferation, apoptosis, or both. They can be activated by structural genetic alterations resulting from mutation or gene fusion. For instance, studies of cytogenetics and allelotyping on fresh tumours and cancer cell lines revealed that allelic losses, genetic mutations, and deletions have been found in the 3p21.3 region, which suggests that one or more putative genes (tumour suppressor genes) were being mutated and leading to various types of cancer [23]. Initiation of most types of cancer is mainly due to the functional loss of a tumour-suppressor gene, followed by alterations in oncogenes and additional tumour-suppressor genes [24]. This, in essence, forms the basis of the multihit hypothesis of cancer initiation.

\section{Tumour Angiogenesis}

The mechanism which involves the growth of new blood vessels from the preexisting blood vessels is called angiogenesis. Via the action of protein factors produced from a tumour in a hypoxic environment, endothelial cells of preexisting 


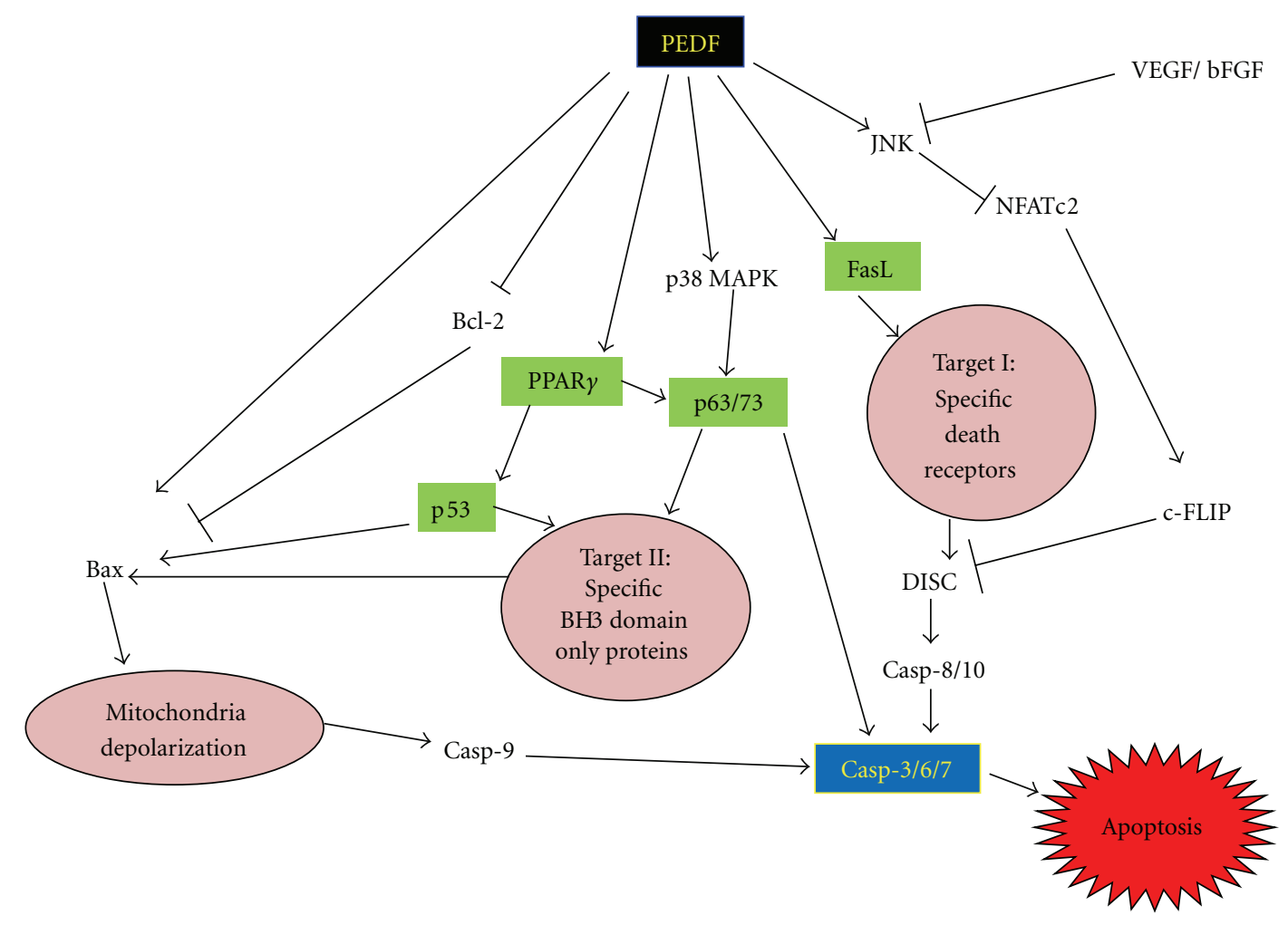

FIGURE 2: PEDF apoptotic pathways. This schematic chart of apoptotic pathways represents concise information from various sources [5, 1417]. PEDF apoptotic pathways. PEDF has been noted to induce apoptosis in mammalian cells via these pathways. It can directly stimulate Bax activity, inhibit Bcl-2, signal through PPAR $\gamma$ to activate p53, signal through p38, FasL, and finally through JNK to block c-FLIP. All these pathways culminate in apoptosis.

capillaries acquire a tip cell phenotype [25]. Movement of endothelial tip cells is directed towards incremental levels of growth factors such as VEGF (vascular endothelial growth factor), which has a triple-pronged role:

(1) triggering the permeability of the capillaries and activation of the tip cell phenotype,

(2) promoting migration of tip cells, and

(3) promoting the proliferation and survival of the stalk ECs.

Migration of tip cells is accompanied by the production of extracellular MMPs (matrix metalloproteinases) which are responsible for the remodeling of the nearby ECM (extracellular matrix). It affects the affinity of VEGF species at different extracellular locations. VEGFs have variety of isoforms which are attached to negatively charged molecules in the ECM, and MMPs somewhat control the balance of these VEGF species. For the creation of a functional capillary network and for the determination of specific vascular patterns, the presence of isoforms with diverse capabilities is essential. After generation of a path by the tip cells in the ECM, in order to form a new lumen for blood circulation, a reorientation of the proliferating ECs is required, in precise coordination with pericytes and other stromal components [25]. Further processes such as anastomosis (linkage of different branches on the network), the action of pressure forces and the intrinsic mechanical properties of the tissue, contribute to the formation of the new vessel network and are finely tuned to determine vascular patterning [26]. Hypoxiainducible factor-1 (HIF-1) production leads to increased VEGF transcription, which causes increased vessel permeability and EC migration and proliferation.

Apart from VEGF, there are many other growth factors responsible for angiogenesis, but VEGF is the most important of all the factors. Hypoxia occurs as the tumour outgrows its existing vascular supply [27]. HIF-1 (hypoxiainducible factor 1) production only leads to increased production of other proangiogenic molecules such as plateletderived growth factor (PDGF), transforming growth factors (TGFs) alpha and beta, basic fibroblast growth factor (bFGF), angiotensin, ephrins, thrombospondin-1 (TSP-1), and a class of protein growth factors called angiopoietins (Ang) $[28,29]$. VEGF administration can initiate vessel formation in adult animals, but by itself promotes formation of only leaky, immature and unstable vessels. In contrast, Ang1 administration seemingly further stabilises and protects the adult vasculature, making it resistant to the damage and leak induced by VEGF or inflammatory challenges.

bFGF, a member of the fibroblast growth factor family plays diverse roles during embryonic development in regulation of cell proliferation, migration [30], and differentiation. 
Angiopoietin-1(Ang-1) [31] plays a significant role at a later stage of blood vessel formation. PDGF plays an important role in normal tissue growth and the pathophysiological processes of vascular diseases like atherosclerosis and restenosis [32]. During the initiation and progression of atherosclerosis, VSMCs are activated by growth factors like PDGF or cytokines, then proliferate and migrate from the media into the intimal surface of the vessel, thus facilitating neointimal formation.

TSP-1 is the first naturally occurring angiogenic inhibitor, which is a multifunctional ECM protein. Downregulation of TSP-1 causes tumour growth alteration by modulating angiogenesis in various types of tumours. It plays a critical role in inhibiting angiogenesis, resulting in the suppression of tumour growth and experimental metastasis [33]. The TGF- $\beta$ pathway occupies a central position in the signaling networks that control growth and differentiation. TGF- $\beta$ is known to have pleiotropic effects which differ according to cell state and differentiation. This includes regulation of proliferation and apoptosis, and stimulation of epithelial-mesenchymal transition (EMT) which together is critical for the development of invasive and metastasis potential [34].

Angiostatin (38 KDa), an internal fragment of plasminogen, is a specific inhibitor of EC proliferation. Akin to angiostatin, researchers isolated an angiogenesis inhibitor named endostatin, a $20 \mathrm{kDa}$ carboxyl-terminal fragment of collagen XVIII. It is a specific inhibitor for endothelial cell proliferation and with systemic therapy, causes suppression of tumour-induced angiogenesis and inhibits tumour growth [35]. It has been shown that when a combination of angiostatin and endostatin gene-encoded proteins are delivered to tumour cells by gene transfer, they can exhibit synergistic antiangiogenic and antitumour effects [36].

\section{Cell Cycling and Apoptosis in Cancer}

The cell cycle plays a significant role in managing to maintain multicellular organisms healthy and alive, in which the parent cell genome undergoes replication and the cell physically divides into daughter cell via cytokinesis. However, genomic stability and cancer may occur due to unwanted mutations caused by some environmental and physiological events. Cells have evolved certain checkpoints to prevent these types of mutations to daughter cells and allow DNA repair mechanism. These checkpoints are controlled by ATM (ataxia telangiectasia mutated) and ATR (ataxia telangiectasia and Rad3-related protein) kinases which activate downstream effector kinases Chk1 (checkpoint kinase 1) and Chk2, respectively. Checkpoints will repair damages in most cases, however if the repair mechanisms cannot repair the DNA damage, cells are either permanently withdrawn from the cell cycle or are eliminated by programmed cell death (apoptosis). Cells may reenter the cell cycle in a process called checkpoint recovery [37]. It is known that programmed cell death, that is, apoptosis, is required for normal development of multicellular organisms, whereby unwanted cells are eliminated during physiological and certain pathological conditions.

Apoptosis is a programmed cell death event which occurs during embryogenesis, metamorphosis, endocrinedependent tissue atrophy, and normal tissue turnover. In multicellular organisms, it is responsible for development, tissue homeostasis, and the immune response via two pathways [38]. They are (i) an intrinsic pathway, which is initiated when the cell is severely damaged, and (ii) the extrinsic pathway, activated when extracellular death ligands are bound by their cognate membrane-associated death receptors.

However, if there is a fault in apoptosis it leads to number of serious diseases including cancer, autoimmunity, and neurodegeneration [39]. When genetically unstable cancer cells tend to acclimatise to a tissue microenvironment that is distant from the primary tumour, a process called metastasis occurs [40]. Metastasis requires the cancer cells to detach from the primary tumour mass, move to and invade blood vessels, survive within the circulation, attach to the endothelium of distant organs, penetrate the endothelial barrier, and establish new tumour colonies. It is this multistage process which is the primary cause of cancer-related deaths, with the primary tumour often resectable. PEDF is responsible for apoptosis of ECs either through the p38/MAPK pathway [14] or through the Fas/FasL pathway [41]. (Detailed PEDFmediated apoptotic pathways are provided in Figure 2).

\section{PEDF and Antiangiogenesis}

Angiogenesis is the process by which new blood vessels are formed from a preexisting microvascular network, and it plays a major role in tumour growth and metastasis. For evaluating antitumour efficacy, Yang et al. [42] constructed PEDF expressing-adenovirus (Ad-PEDF), and with successful transfer of the PEDF gene, antitumour efficacy in a mouse tumour model was tested. The tumour volume was inhibited $\left(2195.1 \pm 462.9 \mathrm{~mm}^{3}\right.$ approximately $\left.50 \%\right)$ with Ad-PEDF when compared to the controls. The mouse which was inoculated with Ad-PEDF showed a 50\% survival rate at day 38, than controls ( $50 \%$ on day 13 ). By using TUNEL (terminal deoxynucleotidyl transferase dUTP nick end labeling) assay, it was determined that the apoptosis index in tumour tissue was higher $(26.3 \% \pm 3.3 \%)$ in the Ad-PEDF group than control groups $(6.3 \% \pm 4.7 \%$ and $5.6 \% \pm 1.9 \%)$, confirming that increased apoptosis caused the accompanying decreased tumour volume. For elucidating whether the increase in apoptosis of Ad-PEDF-treated tumour tissue was associated with the antiangiogenic effect of PEDF, scientists analysed microvessel density (MVD) of tumour tissues and found that Ad-PEDF demonstrated significant decrease in MVD when compared to control groups.

In [43] determined the growth characteristics of a human malignant melanoma cell line overexpressing human PEDF in vitro and in vivo. In contrast to the empty vectortransfected group, PEDF overexpression completely inhibited the growth of the tumour. TUNEL assay was performed to determine the extent of apoptosis in the PEDF-transfected 
and control vector-transfected tumours and it was found that apoptotic cells increased $30 \%$ in the tumours derived from the PEDF-overexpressing G361 cells, when compared with control vector-transfected tumour cells.

In this study, Garcia et al. [44] determined the results of overexpression of PEDF on both melanoma primary tumour growth and metastasis development. Overexpression by melanoma cells inhibited subcutaneous tumour formation. After tail vein injection, PEDF-overexpressing human melanoma cells were unable to grow and metastasise to the lung and liver. The authors also determined the effect of PEDF on proliferation and apoptosis in the subcutaneous tumours and found tumour growth collapse, likely due to impaired vascularisation. PEDF-positive melanoma cells showed significantly lower chemotaxis in a modified Boyden chamber assay. Quantitative data analysis showed a 3- to 5 -fold reduction in migration and invasiveness of PEDF positive cells.

A metabolite isolated from ginseng protopanaxadiol saponins called compound K (CK; 20-O-beta-D-glucopyranosyl-20(S)-protopanaxadiol) has an ability to induce apoptosis in various types of cancers. Based on this, Jeong et al. [45] evaluated the antiangiogenic effects of CK in vitro and in vivo. bFGF induces angiogenesis in human umbilical vein ECs (HUVECs). In vitro tests determined that CK inhibited bFGF-induced proliferation, migration and tube formation. In vivo antiangiogenic activity of CK was evaluated by a Matrigel plug assay and it was found that CK inhibited bFGF-induced angiogenesis. By measuring the levels of VEGF and PEDF, Jeong et al. evaluated the effect of CK on the phosphorylation of p38/MAPK, and AKT in bFGF-treated HUVECs and found decreased levels of VEGF and increased levels of PEDF following CK treatment. These findings suggest that there is a relationship between PEDF, VEGF, and bFGF with respect to angiogenesis.

\section{PEDF and Tumour Cell Differentiation}

Angiogenesis underlies the processes of bone growth, repair, and remodelling and may account at least in part for the aggressive nature of osteosarcoma [46]. PEDF regulates angiogenesis to underlie the physiological processes of bone formation, growth, and remodelling [47]. An elegant balance exists between proangiogenic and antiangiogenic factors in bone so that the degree of vascularisation is appropriate for the biology required. VEGF is widely regarded as the most important proangiogenic factor, while PEDF has been identified as the most-potent antiangiogenic factor [18]. The balance between these two factors is as important in bone physiology as it is in other tissues.

PEDF was found to be expressed in the zones of active bone formation and its role in cell differentiation as well as the maintenance of high growth rates in the mesenchymal cell layer was determined by Lord et al. [48] using a deer antler model. Tombran-Tink and Barnstable hypothesized about the significance of PEDF in bone based on RT-PCR and western blot analysis [14], and more recent studies [49] demonstrated that PEDF inhibits osteoclast formation, survival and bone resorption function, and effects were abrogated by VEGF.

PEDF expression by osteoblasts and osteoclasts in regions of active bone formation regulates neovascularisation at these sites, a process that underlies bone formation, growth and remodelling. Without a supporting vasculature, bone precursors are unable to localise to those sites intended for bone development [50]. Angiogenesis in the ossification and lower hypertrophic zones is necessary for the continued growth and functioning of osteoblasts and osteoclasts, enabling the replacement of cartilaginous tissue by bone, a process ultimately regulated by a balance between PEDF and VEGF [47]. Using immunohistochemistry and in situ hybridisation, PEDF expression was shown to be largely restricted to the chondrocytes of the avascular resting, proliferative and upper hypertrophic layers of the epiphysis, and to regions of active bone remodelling.

In vitro and in vivo studies have revealed that in the case of osteosarcoma, PEDF can induce both indirect and direct suppression of tumour growth and progression by potent antiangiogenic capability of PEDF targeting tumour vasculature and induction of osteosarcoma cell apoptosis, differentiation, and inhibition of cell cycling, respectively [2, 3]. Osteosarcoma usually arises in the metaphysis of long bones. The epiphyseal plate is found between the epiphysis and metaphysis of long bones, which is a natural barrier to tumour invasion. The resistance of epiphyseal cartilage to osteosarcoma invasion is likely to be due to the differential expression of PEDF and VEGF in the zones of the epiphysis [51].

Metastasis is the foremost problem in the treatment of osteosarcoma. In vitro studies with rPEDF revealed dosedependent reduction in cellular invasion by matrigel assay as well as an increase in cell adhesion to collagen type-1 using the human SaOS-2 cell line [52]. In vivo studies done on an orthotopic model [53] showed that treatment with PEDF had the greatest impact on metastases. There was a $70 \%$ reduction in the development of pulmonary metastases and a $40 \%$ reduction in primary tumour size in those mice treated with PEDF [52]. More recently, sustained administration of PEDF via osmotic pumps implanted in mice bearing SaOS-2 tumours demonstrated reduced metastasis to the lungs [54]. Notably, treatment with PEDF was delayed until after the macroscopic appearance of primary tumours. Thus, in this study, both the model used and the delivery regimen were close to the appropriate clinical treatment foreseeable in the future if PEDF is to be used as an osteosarcoma therapeutic.

The critical role of PEDF in regulating MMP activity makes the area of metastasis an intriguing one. Initially, it was found that PEDF can significantly downregulate the levels of MMP-14 but not MMP-2 and -9 in a human chondrosarcoma cell line [55]. This was corroborated by a decreased invasion of cells through collagen-1 matrix in the presence of PEDF. Human melanoma cells were partially inhibited in their invasion, with reduced trafficking of membrane-bound MMP-14 to the cell surface [56].

In [57] demonstrated the ability of PEDF as a multifunctional antitumour agent in neuroblastomas by inhibiting tumour angiogenesis and differentiating tumour cells to 


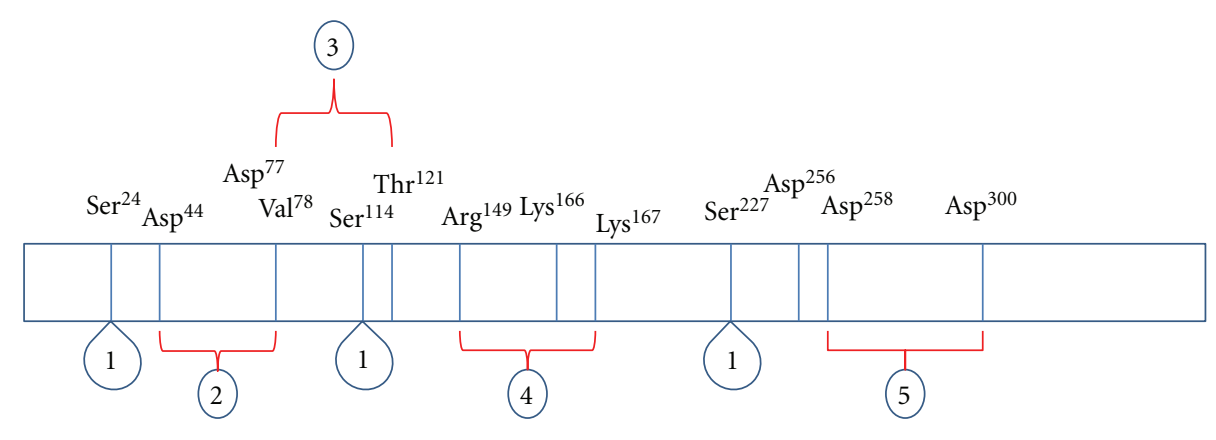

Figure 3: Types of cancers that PEDF has activity against.

produce PEDF. rPEDF cells were injected into the neuroblastoma tumours in vivo. After histological examination, PEDFtreated tumour samples resulted in pale regions corresponding to confluent areas of spindle-shaped cells which are characterised by bland nuclei with abundant cytoplasm. These cells were relatively different from the more primitive neuroblasts observed within the tumour distant from the injection site. The control treatments were composed of undifferentiated neuroblast cells. The authors concluded that even a reduced treatment regimen with PEDF in vivo was capable to promote discrete areas of tumour cell differentiation.

\section{PEDF and Direct Tumour Cell Inhibition}

Most of the human ovarian cancers are derived from the ovarian surface epithelium (OSE), which is made of simple squamous to cuboidal mesothelial cells. In one study, Cheung et al. [58] demonstrated the role of PEDF in tumorigenesis of ovarian cancer in vitro. MTT cell viability and proliferation assay were performed to determine the physiological functions of PEDF. The treatment with recombinant PEDF ( $\mathrm{rPEDF}$ ) resulted in a dose-dependent decrease in cell growth and concomitant increase in apoptosis, when compared with controls. The role of endogenous PEDF in the regulation of cell proliferation and viability was determined by RNA interference. This study is the first to explain the role of PEDF in OSE biology and ovarian cancer and suggested that the loss of PEDF may be of relevance in carcinogenesis.

In another study, human pancreatic adenocarcinoma cell lines were stably transfected with PEDF [59], and the effects of overexpression investigated in a murine subcutaneous tumour model. Overexpression with a lentivirus-PEDF (LVPEDF) vector resulted in 28 and $61 \%$ inhibition of proliferation and migration of cells, respectively. PEDF overexpression was evaluated in vivo and treatment significantly suppressed tumour growth and peritoneal metastasis.

PEDF can suppress osteosarcoma growth, angiogenesis, and metastasis by its multitargeted antitumour activities both in vitro and in vivo [51]. The in vitro anti-proliferative effect was evaluated on two osteosarcoma cell lines rat UMR 106-01 and human SaOS-2 and resulted in dose-dependent reduction in cell proliferation. Varying concentrations of rPEDF were used to determine whether rPEDF induced apoptosis in osteosarcoma, and then analysed by TUNEL assay to determine the percentage of cells undergoing apoptosis and found that rPEDF significantly increased apoptosis and suppressed proliferation of cells. An orthotopic SaOS2 tumour model [53], consisting of cells injected into the proximal tibiae of nude mice and co-administered with rPEDF showed a significant reduction in primary tumour size, growth rates and a significant reduction in pulmonary metastases.

Osteosarcoma, a prominent primary bone tumour, represents the second highest cause of cancer-related death in childhood and adolescence. Ek et al. [52] demonstrated the potential of plasmid mediated gene transfer of PEDF for direct inhibition of tumour growth, angiogenesis and metastasis in two clinically relevant orthotopic models of osteosarcoma (rat UMR 106-01 and human SaOS-2) via both in vitro and in vivo. The team examined whether PEDF overexpression influences in vivo primary tumour growth and the development of pulmonary metastasis. UMRPEDF (PEDF overexpression) tumours demonstrated slower growth kinetics compared to the parental and vector groups; with a two- to threefold reduction in tumour growth and $\mathrm{SaOS}_{\mathrm{PEDF}}$ tumours were 59\% smaller, respectively. While all mice in the control groups developed lung metastases, only $20 \%$ of mice with UMR PEDF $_{\text {fumours and no mice with SaOS }}$ PEDF tumours developed pulmonary metastases. Immunohistochemical staining of the tumour microvascular ECs with an antibody against CD34 evaluated that a significant reduction in MVD (microvessel density) in both PEDF-overexpressing tumour groups with an 85 and $74 \%$ decrease seen in the $\mathrm{UMR}_{\mathrm{PEDF}}$ and $\mathrm{SaOS}$ PEDF groups, respectively.

PEDF is known as a multifunctional protein which possesses potent antiangiogenic, neurotrophic, neuroprotective, immunosuppressive, and antitumorigenic properties (see Figure 3). Ek et al. [60] identified potential functional epitopes on the PEDF protein sequence and determined their antitumour activity in the human osteosarcoma cell line SaOS-2. They characterized the bioactivity of four synthetic peptides corresponding to sequences 40-64 (StVOrth-1), 78-102 (StVOrth-2), 90-114 (StVOrth-3), and 387-411 (StVOrth-4) of human PEDF using SaOS-2. They evaluated the antiproliferative effects of the peptides and found that StVOrth-2 exhibited the most significant anti-proliferative activity, with a reduction of $59 \%$ and $63 \%$ seen at day 5 with lower $(5 \mathrm{nM})$ and higher $(25 \mathrm{nM})$ concentrations, respectively. They examined the potential behind suppression of 
tumour cell invasion through Matrigel and found that all four peptides significantly decreased Matrigel invasion by greater that 50\%. However StVOrth-4 provided that most consistent inhibition, with greater than $70 \%$ reduction observed at all concentrations. Ek et al. examined treatment with the PEDF-derived peptides for understanding the alteration of osteosarcoma cell adhesion to collagen (a potential mechanism in antimetastasis) and found that all four peptides considerably increased cell adhesion to collagen type 1 , with StVOrth-3 demonstrating 53\% greater ability than the other peptides. Prodifferentiation potential studies of the PEDF fragments in vitro revealed that administration of StVOrth-1, -2, and -3 significantly increased nodule formation in SaOS-2 cells. They further tested the in vivo activity of StVOrth-2 and StVOrth-3 in an orthotopic model of human osteosarcoma, using a model employing tumour cells premixed with peptides, then injected orthotopically. StVOrth-2 exhibited predominantly antiproliferation activity, while StVOrth-3 caused mainly increased collagen adhesion and VEGF suppression. The results of an efficacy study using sustained delivery of peptides in the orthotopic model of osteosarcoma is included in this issue $[5,54]$.

In a more recent study, Mirochnik et al. [61] have demonstrated improved antiangiogenic activity by the small PEDF peptide, only $18 \mathrm{mer}$ in length, called P18. P18 inhibited the growth of prostate tumour growth and the more aggressive Renca cells in vivo in the subcutaneous xenograft model at $10 \mathrm{mg} / \mathrm{kg}$. At the same dose, the 34-mer had no significant effect on tumour growth. The authors stated that P18 was more potent at blocking tumor angiogenesis and causing higher levels of intratumoral apoptosis.

\section{Future Directions}

PEDF is a promising therapeutic agent for various types of cancers. Until now it is known that it is capable of reducing cancer via inhibiting angiogenesis, tumour growth, cell migration and inducing apoptosis and tumour cell differentiation. Only a little is known about the proangiogenic factors like VEGF, bFGF, PDGF, and there is lot to be known about other proangiogenic factors that interact with PEDF. The fact that regions of PEDF have different functional activity may lead to synthesis of small and cheap peptides which can be tested as anticancer drugs in the near future. Recent biochemical studies have provided some insight into PEDF structure and function. However, very few articles have looked at PEDF receptor-mediated signalling in cancer. Some receptors (G-protein-coupled receptor GPR39) can protect cells from death by increasing secretion of PEDF [62]. More work has to be done in this area given that more than two receptors for this protein have been found to date.

\section{Summary}

Cancer is characterized by uncontrolled growth and spread of abnormal cells. PEDF is known to be a multifunctional protein which possesses potent antiangiogenic, neurotrophic, neuroprotective, immunosuppressive, and antitumorigenic properties. Scientists designed Ad-PEDF for evaluating the antitumour efficacy and demonstrated role of PEDF in apoptosis and angiogenesis. Other group of researchers demonstrated that overexpression of PEDF suppressed tumour growth and angiogenesis and enhanced the rate of apoptosis. PEDF regulation of angiogenesis underlies the physiological processes of bone formation, growth and remodelling. PEDF can induce both indirect and direct suppression of tumour growth and progression by potent antiangiogenic capability. An orthotopic model of osteosarcoma studies showed that treatment with PEDF had the greatest impact on metastases. There is now justification for evaluation of PEDF efficacy in other types of cancers.

\section{Conflict of Interests}

The authors declare that there is not conflict of interests in writing this paper.

\section{Acknowledgments}

The authors acknowledge funding from the Victoria University Research Development Grant Scheme (VU-RDGS) and an ANZ Trustees/Medical Research and Technology in Victoria Grant to C. Dass.

\section{References}

[1] American Cancer Society, 2010, http://www.cancer.org /Research/CancerFactsFigures/CancerFactsFigures/index.

[2] E. T. H. Ek, C. R. Dass, and P. F. M. Choong, "Pigment epithelium-derived factor: a multimodal tumor inhibitor," Molec ular Cancer Therapeutics, vol. 5, no. 7, pp. 1641-1646, 2006.

[3] E. T. H. Ek, C. R. Dass, and P. F. M. Choong, "PEDF: a potential molecular therapeutic target with multiple anticancer activities," Trends in Molecular Medicine, vol. 12, no. 10, pp. 497-502, 2006.

[4] A. R. Murray and J. X. Ma, "PEDF as a treatment for cervical cancer," Cancer Biology and Therapy, vol. 9, no. 12, pp. 975977, 2010.

[5] M. L. Broadhead, C. R. Dass, and P. F. Choong, "In vitro and in vivo biological activity of PEDF against a range of tumors," Expert Opinion on Therapeutic Targets, vol. 13, no. 12, pp. 1429-1438, 2009.

[6] J. Yang, E. J. Duh, R. B. Caldwell, and M. Ali Behzadian, "Antipermeability function of PEDF involves blockade of the MAP kinase/GSK/ $\beta$-catenin signaling pathway and UPAR expression," Investigative Ophthalmology and Visual Science, vol. 51, no. 6, pp. 3273-3280, 2010.

[7] S. Halin, P. Wikström, S. H. Rudolfsson et al., "Decreased pigment epithelium-derived factor is associated with metastatic phenotype in human and rat prostate tumors," Cancer Research, vol. 64, no. 16, pp. 5664-5671, 2004.

[8] H. Uehara, M. Miyamoto, K. Kato et al., "Expression of pigment epithelium-derived factor decreases liver metastasis and correlates with favorable prognosis for patients with ductal pancreatic adenocarcinoma," Cancer Research, vol. 64, no. 10, pp. 3533-3537, 2004.

[9] K. Matsumoto, H. Ishikawa, D. Nishimura, K. Hamasaki, K. Nakao, and K. Eguchi, "Antiangiogenic property of pigment 
epithelium-derived factor in hepatocellular carcinoma," Hepatology, vol. 40, no. 1, pp. 252-259, 2004.

[10] L. P. Abramson, V. Stellmach, J. A. Doll et al., "Wilms' tumor growth is suppressed by antiangiogenic pigment epitheliumderived factor in a xenograft model," Journal of Pediatric Surgery, vol. 38, no. 3, pp. 336-342, 2003.

[11] J. Tombran-Tink, K. Mazuruk, I. R. Rodriguez et al., "Organization, evolutionary conservation, expression and unusual Alu density of the human gene for pigment epithelium-derived factor, a unique neurotrophic serpin.," Molecular Vision, vol. 2, p. 11, 1996.

[12] S. Patricia Becerra, "Focus on molecules: pigment epitheliumderived factor (PEDF)," Experimental Eye Research, vol. 82, no. 5, pp. 739-740, 2006.

[13] N. Yasui, T. Mori, D. Morito et al., "Dual-site recognition of different extracellular matrix components by antiangiogenic/neurotrophic serpin, PEDF," Biochemistry, vol. 42, no. 11 , pp. 3160-3167, 2003.

[14] O. V. Volpert, T. Zaichuk, W. Zhou et al., "Inducer-stimulated Fas targets activated endothelium for destruction by antiangiogenic thrombospondin-1 and pigment epitheliumderived factor," Nature Medicine, vol. 8, no. 4, pp. 349-357, 2002.

[15] T. A. Zaichuk, E. H. Shroff, R. Emmanuel, S. Filleur, T. Nelius, and O. V. Volpert, "Nuclear factor of activated T cells balances angiogenesis activation and inhibition," Journal of Experimental Medicine, vol. 199, no. 11, pp. 1513-1522, 2004.

[16] T. C. Ho, S. L. Chen, Y. C. Yang, C. L. Liao, H. C. Cheng, and Y. P. Tsao, "PEDF induces p53-mediated apoptosis through PPAR gamma signaling in human umbilical vein endothelial cells," Cardiovascular Research, vol. 76, no. 2, pp. 213-223, 2007.

[17] T. Zhang, M. Guan, C. Xu, Y. Chen, and Y. Lu, "Pigment epithelium-derived factor inhibits glioma cell growth in vitro and in vivo," Life Sciences, vol. 81, no. 16, pp. 1256-1263, 2007.

[18] D. W. Dawson, O. V. Volpert, P. Gillis et al., "Pigment epithelium-derived factor: a potent inhibitor of angiogenesis," Science, vol. 285, no. 5425, pp. 245-248, 1999.

[19] D. Hanahan and J. Folkman, "Patterns and emerging mechanisms of the angiogenic switch during tumorigenesis," Cell, vol. 86, no. 3, pp. 353-364, 1996.

[20] G. Maik-Rachline, S. Shaltiel, and R. Seger, "Extracellular phosphorylation converts pigment epithelium-derived factor from a neurotrophic to an antiangiogenic factor," Blood, vol. 105, no. 2, pp. 670-678, 2005.

[21] A. Castellanos, C. Vicente-Dueñas, E. Campos-Sánchez et al., "Cancer as a reprogramming-like disease: Implications in tumor development and treatment," Seminars in Cancer Biology, vol. 20, no. 2, pp. 93-97, 2010.

[22] S. V. Sharma, D. W. Bell, J. Settleman, and D. A. Haber, "Epidermal growth factor receptor mutations in lung cancer," Nature Reviews Cancer, vol. 7, no. 3, pp. 169-181, 2007.

[23] L. Ji and J. A. Roth, "Tumor suppressor FUS1 signaling pathway," Journal of Thoracic Oncology, vol. 3, no. 4, pp. 327-330, 2008.

[24] C. M. Croce, "Oncogenes and cancer," New England Journal of Medicine, vol. 358, no. 5, pp. 502-511, 2008.

[25] R. D. M. Travasso, E. C. Poiré, M. Castro, J. C. RodrguezManzaneque, and A. Hernández-Machado, "Tumor angiogenesis and vascular patterning: a mathematical model," PLoS One, vol. 6, no. 5, Article ID e19989, 2011.

[26] E. A. V. Jones, F. Le Noble, and A. Eichmann, "What determines blood vessel structure? Genetic prespecification vs. hemodynamics," Physiology, vol. 21, no. 6, pp. 388-395, 2006.
[27] J. A. Forsythe, B. H. Jiang, N. V. Iyer et al., "Activation of vascular endothelial growth factor gene transcription by hypoxia-inducible factor 1," Molecular and Cellular Biology, vol. 16, no. 9, pp. 4604-4613, 1996.

[28] G. D. Yancopoulos, S. Davis, N. W. Gale, J. S. Rudge, S. J. Wiegand, and J. Holash, "Vascular-specific growth factors and blood vessel formation," Nature, vol. 407, no. 6801, pp. 242248, 2000.

[29] R. K. Jain, "Molecular regulation of vessel maturation," Nature Medicine, vol. 9, no. 6, pp. 685-693, 2003.

[30] J. W. Choi, S. Kim, T. M. Kim et al., "Basic fibroblast growth factor activates MEK/ERK cell signaling pathway and stimulates the proliferation of chicken primordial germ cells," PLoS One, vol. 5, no. 9, Article ID e12968, 2010.

[31] G. Thurston, "Complementary actions of VEGF and Angiopoietin-1 on blood vessel growth and leakage," Journal of Anatomy, vol. 200, no. 6, pp. 575-580, 2002.

[32] C. H. Heldin and B. Westermark, "Mechanism of action and in vivo role of platelet-derived growth factor," Physiological Reviews, vol. 79, no. 4, pp. 1283-1316, 1999.

[33] B. Ren, K. O. Yee, J. Lawler, and R. Khosravi-Far, "Regulation of tumor angiogenesis by thrombospondin-1," Biochimica et Biophysica Acta, vol. 1765, no. 2, pp. 178-188, 2006.

[34] S. Sheahan, C. O. Bellamy, S. N. Harland, D. J. Harrison, and S. Prost, "TGFbeta induces apoptosis and EMT in primary mouse hepatocytes independently of p53, p21Cip1 or Rb status," BMC Cancer, vol. 8, article no. 191, 2008.

[35] M. S. O’Reilly, L. Holmgren, Y. Shing et al., "Angiostatin: a novel angiogenesis inhibitor that mediates the suppression of metastases by a Lewis lung carcinoma," Cell, vol. 79, no. 2, pp. 315-328, 1994.

[36] S. Y. Paek, Y. S. Kim, and S. G. Choi, "The orientationdependent expression of angiostatin-endostatin hybrid proteins and their characterization for the synergistic effects of antiangiogenesis," Journal of Microbiology and Biotechnology, vol. 20, no. 10, pp. 1430-1435, 2010.

[37] R. H. Medema and L. Macůrek, "Checkpoint recovery in cells: how a molecular understanding can help in the fight against cancer," F1000 Biology Reports, vol. 3, no. 1, article no. 10, 2011.

[38] M. O. Hengartner, “The biochemistry of apoptosis," Nature, vol. 407, no. 6805, pp. 770-776, 2000.

[39] C. Gu, J. Zhang, Y. Chen, and J. Lei, "A trigger model of apoptosis induced by tumor necrosis factor signaling," $B M C$ Systems Biology, vol. 5, no. 1, article no. 13, 2011.

[40] G. P. Gupta and J. Massagué, "Cancer metastasis: building a framework," Cell, vol. 127, no. 4, pp. 679-695, 2006.

[41] S. X. Zhang, J. J. Wang, G. Gao, K. Parke, and J. X. Ma, "Pigment epithelium-derived factor downregulates vascular endothelial growth factor (VEGF) expression and inhibits VEGF-VEGF receptor 2 binding in diabetic retinopathy," Journal of Molecular Endocrinology, vol. 37, no. 1, pp. 1-12, 2006.

[42] L. P. Yang, P. Cheng, X. C. Peng et al., "Anti-tumor effect of adenovirus-mediated gene transfer of pigment epitheliumderived factor on mouse B16-F10 melanoma," Journal of Experimental and Clinical Cancer Research, vol. 28, no. 1, article no. 75, 2009.

[43] R. Abe, T. Shimizu, S. I. Yamagishi et al., "Overexpression of pigment epithelium-derived factor decreases angiogenesis and inhibits the growth of human malignant melanoma cells in vivo," American Journal of Pathology, vol. 164, no. 4, pp. 12251232, 2004. 
[44] M. Garcia, N. I. Fernandez-Garcia, V. Rivas et al., "Inhibition of xenografted human melanoma growth and prevention of metastasis development by dual antiangiogenic/antitumor activities of pigment epithelium-derived factor," Cancer Research, vol. 64, no. 16, pp. 5632-5642, 2004.

[45] A. Jeong, H. J. Lee, S. J. Jeong et al., "Compound K inhibits basic fibroblast growth factor-induced angiogenesis via regulation of p38 mitogen activated protein kinase and AKT in human umbilical vein endothelial cells," Biological and Pharmaceutical Bulletin, vol. 33, no. 6, pp. 945-950, 2010.

[46] G. M. Quan, J. Ojaimi, and P. F. Choong, "Cartilage-derived anti-angiogenic therapy for osteosarcoma: a novel approach," International Journal of Cancer Prevention, vol. 1, pp. 411-419, 2004.

[47] G. M. Y. Quan, J. Ojaimi, Y. Li, V. Kartsogiannis, H. Zhou, and P. F. M. Choong, "Localization of pigment epithelium-derived factor in growing mouse bone," Calcified Tissue International, vol. 76, no. 2, pp. 146-153, 2005.

[48] E. A. Lord, S. K. Martin, J. P. Gray, C. Li, and D. E. Clark, "Cell cycle genes PEDF and CDKN1C in growing Deer antlers," Anatomical Record, vol. 290, no. 8, pp. 994-1004, 2007.

[49] T. Akiyama, C. R. Dass, Y. Shinoda, H. Kawano, S. Tanaka, and P. F. M. Choong, "PEDF regulates osteoclasts via osteoprotegerin and RANKL," Biochemical and Biophysical Research Communications, vol. 391, no. 1, pp. 789-794, 2010.

[50] G. M. Y. Quan, J. Ojaimi, A. P. W. Nadesapillai, H. Zhou, and P. F. M. Choong, "Resistance of epiphyseal cartilage to invasion by osteosarcoma is likely to be due to expression of antiangiogenic factors," Pathobiology, vol. 70, no. 6, pp. 361$367,2002$.

[51] E. T. H. Ek, C. R. Dass, K. G. Contreras, and P. F. M. Choong, "Pigment epithelium-derived factor overexpression inhibits orthotopic osteosarcoma growth, angiogenesis and metastasis," Cancer Gene Therapy, vol. 14, no. 7, pp. 616-626, 2007.

[52] E. T. H. Ek, C. R. Dass, K. G. Contreras, and P. F. M. Choong, "Inhibition of orthotopic osteosarcoma growth and metastasis by multitargeted antitumor activities of pigment epitheliumderived factor," Clinical and Experimental Metastasis, vol. 24, no. 2, pp. 93-106, 2007.

[53] C. R. Dass, E. T. Ek, K. G. Contreras, and P. F. Choong, "A novel orthotopic murine model provides insights into cellular and molecular characteristics contributing to human osteosarcoma," Clinical and Experimental Metastasis, vol. 23, no. 7-8, pp. 367-380, 2006.

[54] M. L. Broadhead, C. R. Dass, and P. F. M. Choong, "Systemically administered PEDF against primary and secondary tumours in a clinically relevant osteosarcoma model," British Journal of Cancer, vol. 105, no. 10, pp. 1503-1511, 2011.

[55] M. L. Tan, P. F. M. Choong, and C. R. Dass, "Antichondrosarcoma effects of PEDF mediated via molecules important to apoptosis, cell cycling, adhesion and invasion," Biochemical and Biophysical Research Communications, vol. 398, no. 4, pp. 613-618, 2010.

[56] O. Ladhani, C. Sánchez-Martinez, J. L. Orgaz, B. Jimenez, and O. V. Volpert, "Pigment epithelium-derived factor blocks tumor extravasation by suppressing amoeboid morphology and mesenchymal proteolysis1,2," Neoplasia, vol. 13, no. 7, pp. 633-642, 2011.

[57] S. E. Crawford, V. Stellmach, M. Ranalli et al., "Pigment epithelium-derived factor (PEDF) in neuroblastoma: a multifunctional mediator of Schwann cell antitumor activity," Journal of Cell Science, vol. 114, no. 24, pp. 4421-4428, 2001.
[58] L. W. T. Cheung, S. C. L. Au, A. N. Y. Cheung et al., "Pigment epithelium-derived factor is estrogen sensitive and inhibits the growth of human ovarian cancer and ovarian surface epithelial cells," Endocrinology, vol. 147, no. 9, pp. 4179-4191, 2006.

[59] R. Hase, M. Miyamoto, H. Uehara et al., "Pigment epitheliumderived factor gene therapy inhibits human pancreatic cancer in mice," Clinical Cancer Research, vol. 11, no. 24, pp. 87378744, 2005.

[60] E. T. H. Ek, C. R. Dass, K. G. Contreras, and P. F. M. Choong, "PEDF-derived synthetic peptides exhibit antitumor activity in an orthotopic model of human osteosarcoma," Journal of Orthopaedic Research, vol. 25, no. 12, pp. 1671-1680, 2007.

[61] Y. Mirochnik, A. Aurora, F. T. Schulze-Hoepfner et al., "Short pigment epithelial-derived factor-derived peptide inhibits angiogenesis and tumor growth," Clinical Cancer Research, vol. 15, no. 5, pp. 1655-1663, 2009.

[62] S. Dittmer, M. Sahin, A. Pantlen et al., "The constitutively active orphan G-protein-coupled receptor GPR39 protects from cell death by increasing secretion of pigment epitheliumderived growth factor," Journal of Biological Chemistry, vol. 283, no. 11, pp. 7074-7081, 2008. 

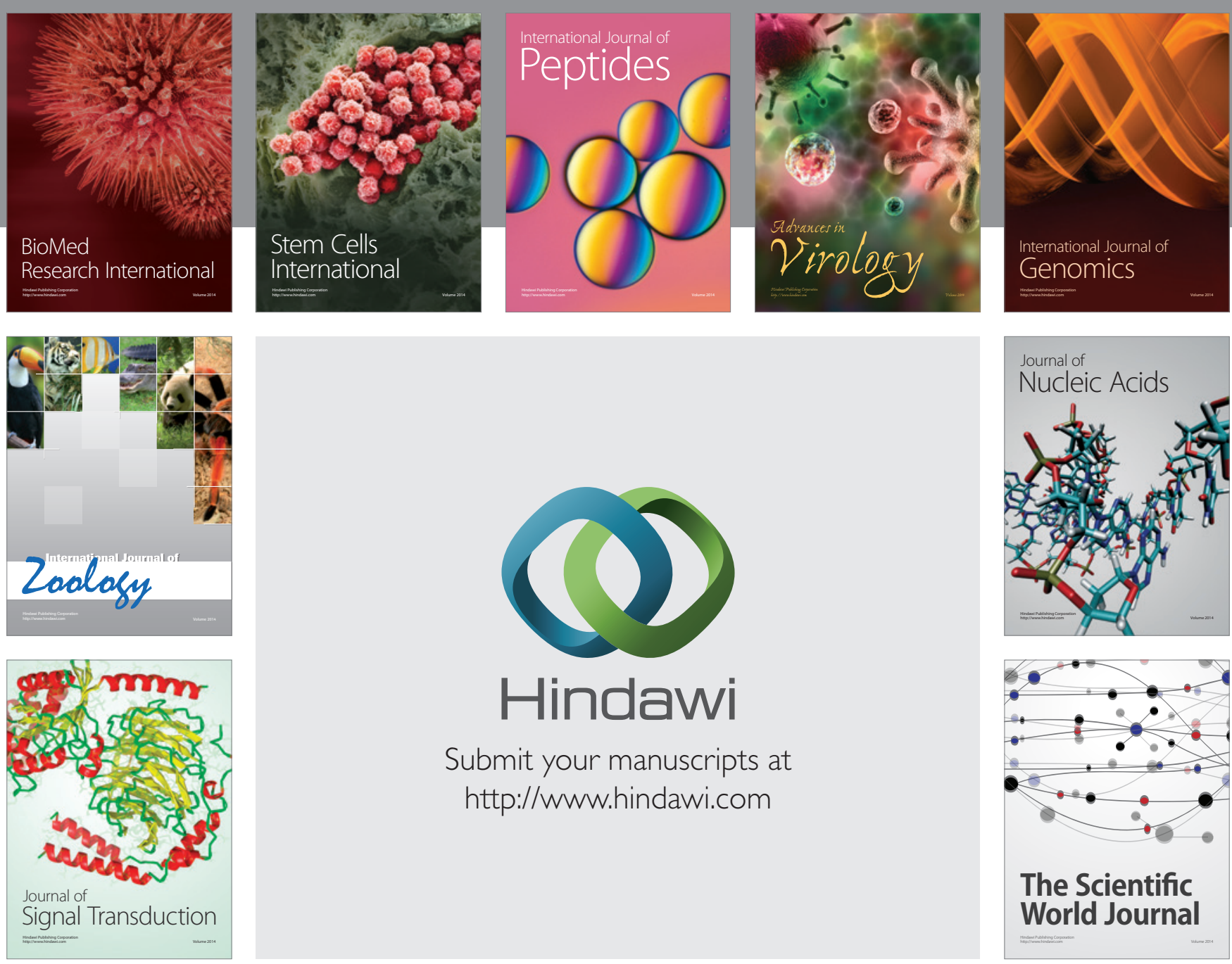

Submit your manuscripts at

http://www.hindawi.com
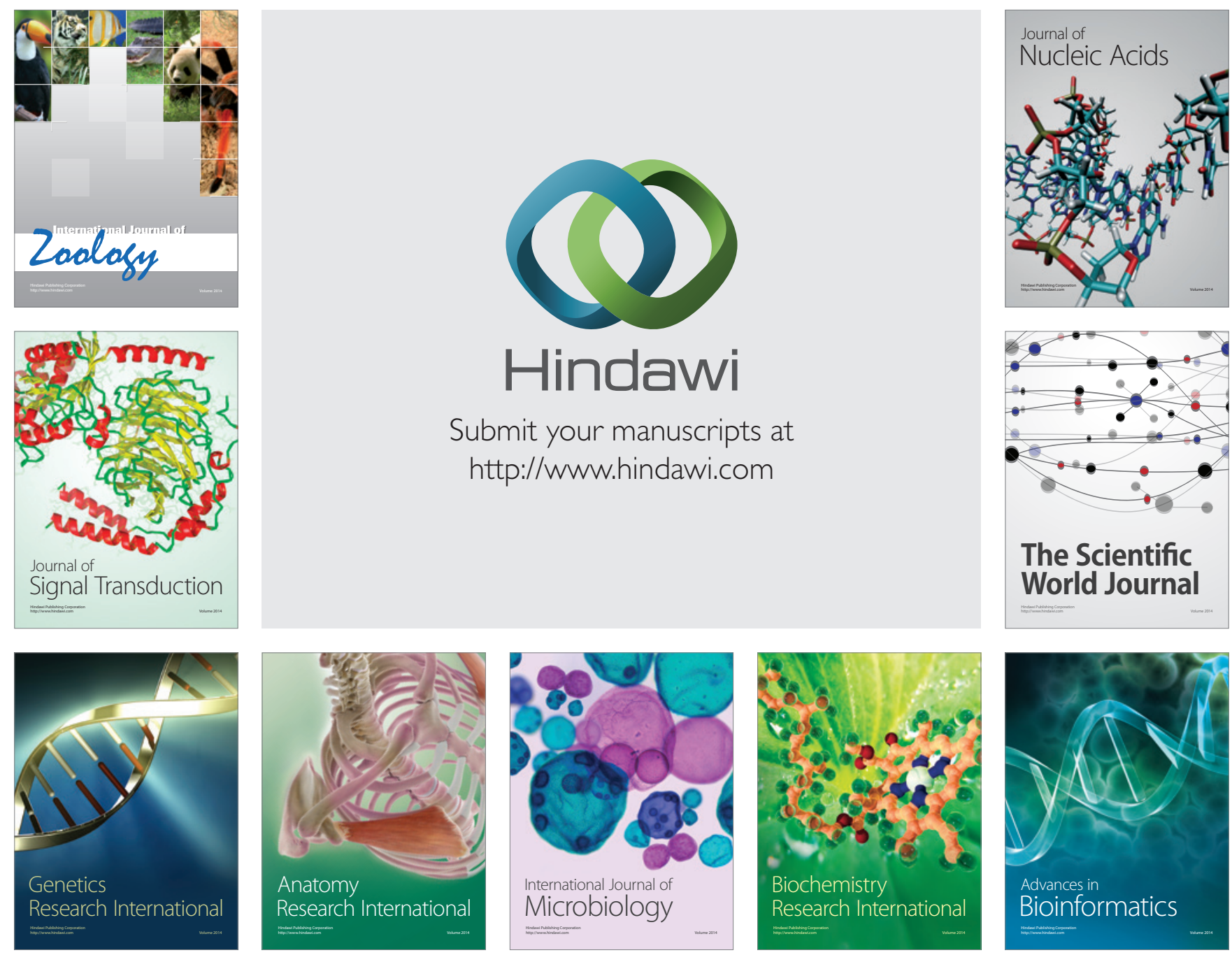

The Scientific World Journal
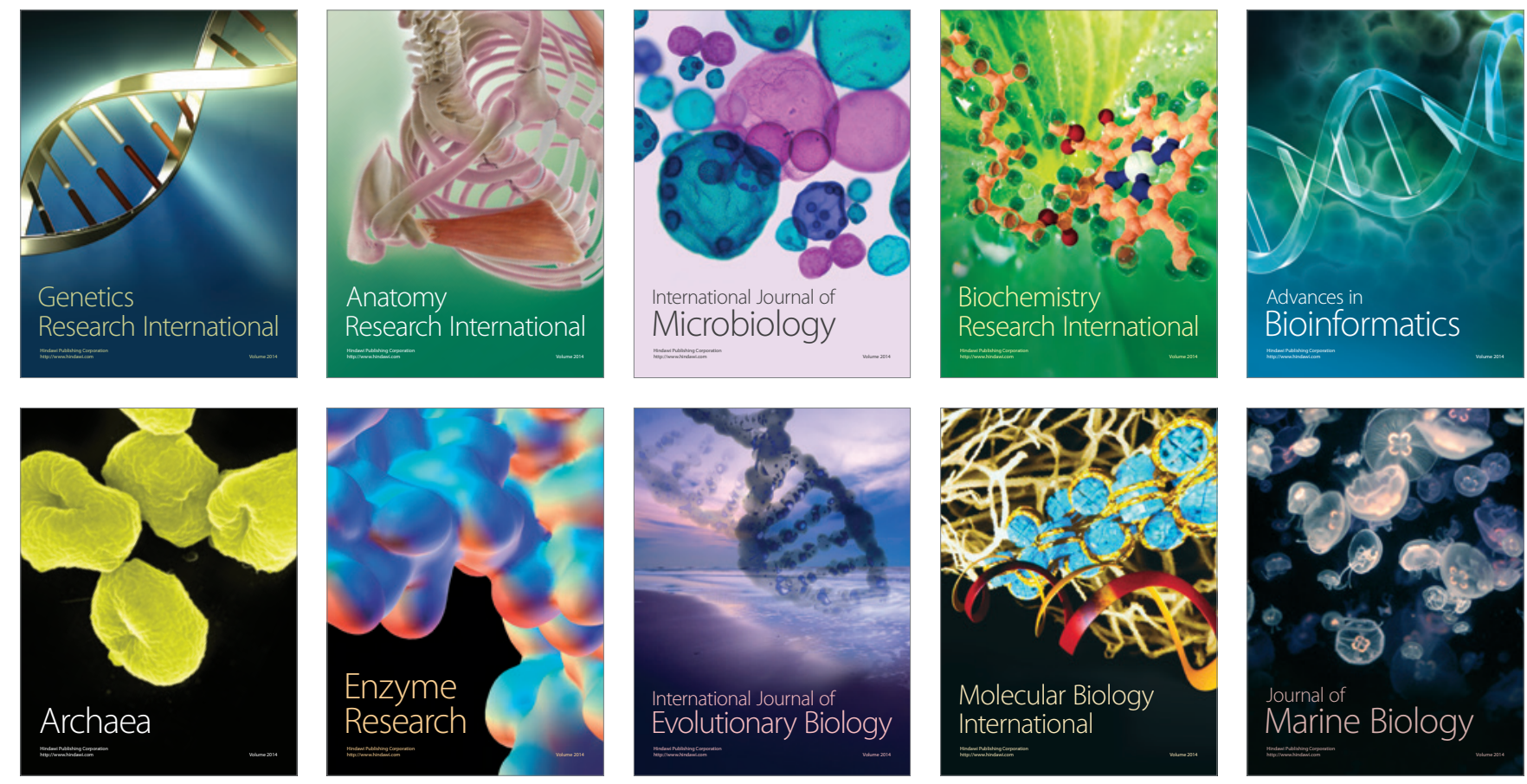